\title{
LA EDUCACIÓN EN TIEMPOS DE GLOBALIZACIÓN: ¿QUIÉN SE BENEFICIA?
}

XAVIer BOnaL

\begin{abstract}
RESUMEN: Las políticas educativas desarrolladas en el contexto del auge del neoliberalismo económico asumen una serie de efectos beneficiosos para el crecimiento económico y la igualdad de oportunidades educativas. Su hegemonía a lo largo de los últimos años, con un especial protagonismo del Banco Mundial, se ha traducido en su extensión en diversas partes del globo, y especialmente en los países en vías de desarrollo. Dichas políticas se presentan como respuesta a los retos de la globalización y como estrategia eficaz para asegurar beneficios colectivos del desarrollo educativo, especialmente para aquellos grupos socialmente más desfavorecidos. El artículo identifica las principales tesis de la política educativa neoliberal en el terreno de las relaciones entre educación y empleo, entre expansión educativa y gasto público y en las estrategias educativas de lucha contra la pobreza. Para cada una de ellas cuestiona los efectos esperados de las propuestas políticas hegemónicas y ofrece evidencias de resultados que muestran que en ningún caso los beneficiarios son los colectivos más desfavorecidos.
\end{abstract}

Palabras clave: Globalización. Desarrollo. Política educativa. Pobreza. Desigualdad.

\section{EDUCATION IN THE ERA OF GLOBALISATION: WHO BENEFITS?}

ABSTRACT: In the context of economic neoliberalism, educational policies assume positive effects for both economic growth and equality of educational opportunities. In recent years, this thinking has been hegemonic: it has been extended around the world and especially in developing countries (with a special role of the World

Doctor en Sociología y profesor del Departamento de Sociología de la Universitat Autónoma de Barcelona (España).E-mail: xavier.bonal@uab.cat

Educ. Soc., Campinas, vol. 30, n. 108, p. 653-671, out. 2009 
Bank). Those policies are presented as a response to the challenges of globalisation and as the correct strategy to ensure collective benefits of educational development, especially for socially disadvantaged groups. This paper identifies the main theses of neoliberal educational policy. The relationship of education and labor market, the connection between educational expansion and public expenditure and educational strategies to combat poverty are explored as some of the fields where neoliberal educational policies are produced. Expected effects of educational policy measures are questioned for each of these fields. At the same time, the text provides evidence of results that show that the most disadvantaged groups are not those that can really obtain the main benefits of mainstream educational policies.

Key words: Globalisation. Development. Educational policy. Poverty. Inequality.

\section{Introducción}

G

lobalización" ha sido sin duda uno de los términos más utilizados en las ciencias sociales a lo largo de las últimas décadas. A pesar de la intensidad de su uso, sin embargo, no parece existir consenso acerca de la precisión del concepto. La diversidad y dispersión terminológica tiene relación tanto con la multiplicidad de factores que pueden explicarla (revolución de las tecnologías de la información y la comunicación, final de la Guerra Fría y establecimiento de un nuevo orden internacional, auge de las doctrinas neoliberales etc.), como con el enfoque adoptado para evaluar sus implicaciones (globalización económica y financiera, globalización política o globalización cultural). Proyectar las consecuencias de esta complejidad sobre diferentes políticas e instituciones sociales no es pues una tarea que pueda resolverse con simplicidad: exige forzosamente una aproximación pluridisciplinar e imaginación metodológica para traducir la ambigüedad conceptual en propuestas concretas de análisis.

Las múltiples dimensiones del concepto y la diversidad de efectos asociados a aquello que denominamos "globalización" deberían tener como consecuencia la cautela respecto a las supuestas relaciones causaefecto de los fenómenos sociales. Los cambios asociados a procesos de 
globalización difícilmente permiten identificar relaciones unívocas entre variables. Valgan como ejemplos el hecho de que, en contextos de procesos de "globalización", la tecnología no siempre destruye puestos de trabajo o no siempre los crea, el aumento del comercio internacional no siempre se traduce en crecimiento económico, la descentralización de la gestión pública no siempre mejora las condiciones de vida del ciudadano, o la mayor circulación de mercancías culturales no se traduce inequívocamente en mejorar el nivel cultural de la población.

Sin embargo, la complejidad no ha sido en absoluto obstáculo para la emergencia de axiomas aparentemente incuestionables acerca de determinadas relaciones causa-efecto, una vez la globalización económica, política y cultural se constituye en marco explicativo fundamental de los fenómenos sociales. La reducción de las relaciones causa-efecto en un único sentido forma parte de una ideología que minimiza la complejidad de las relaciones sociales y genera formas de pensamiento único respecto a las virtudes de determinadas políticas. En las últimas décadas hemos podido observar los componentes de esta ideología en todos los terrenos de la vida social. Lejos de ser estáticos, los discursos dominantes han mostrado capacidad de adaptación ante una evidencia empírica que se resiste a confirmar sus tesis. Piénsese por ejemplo en las transformaciones experimentadas por el Consenso de Washington, a medida que las fórmulas de política económica asociadas al mismo mostraban sus efectos sobre las condiciones de vida de la población. A pesar de que el consenso dio lugar al post-consenso, nunca se "permitió" a los científicos sociales abandonar realmente el "acuerdo" acerca de las "imperfecciones" que motivaron su fracaso (Fine, 2001).

La hegemonía acerca de las mejores políticas para hacer frente a los retos de la globalización ha alcanzado a todos los ámbitos del sector público. La educación, por supuesto, no sólo no ha sido una excepción sino un espacio privilegiado de producción del discurso hegemónico. Sostengo que existen dos razones fundamentales para explicarlo. Por una parte, la educación es un componente fundamental para la construcción de las llamadas "sociedades del conocimiento", y son estas formaciones sociales las que pueden obtener la mayor ventaja comparativa ante la globalización económica. La producción de 
conocimiento pasa a ser no sólo un medio, sino un fin en sí mismo del proceso de desarrollo, con lo que la inversión educativa estratégica se convierte en fundamental para el éxito en contextos de elevada competitividad internacional. Por otra parte, la globalización también alcanza a la educación como mercancía. La agenda de la Organización Mundial del Comercio (OMC) y, concretamente, el Acuerdo General sobre el Comercio de Servicios (AGCS) sitúan a la educación como servicio comercializable extraordinariamente atractivo para el capital, con lo que las presiones acerca del "proteccionismo" del Estado en educación son cada vez mayores y se activan discursos acerca de los beneficios de la globalización de la provisión de servicios educativos (Robertson, Bonal y Dale, 2002; Verger, 2008).

Uno de los aspectos más significativos de la producción de discursos y políticas educativas dominantes es identificable en los supuestos beneficios universales de las reformas educativas. Si bien es la globalización lo que exige tales reformas, el cambio debe entenderse siempre beneficioso para todos, y especialmente para los grupos sociales más desfavorecidos. En efecto, cualquiera de las reformas que forman parte de la agenda educativa global, como veremos, identifica ventajas directas o indirectas sobre los grupos sociales más excluidos o vulnerables. Los efectos potencialmente perjudiciales para dichos grupos, en cambio, tienden a ser minimizados.

Las páginas siguientes se centran en algunas de las tesis que estructuran la agenda educativa global para el desarrollo. Constituyen las bases de lo que determinados autores señalan como reglas de juego establecidas por agentes del proceso de globalización en educación (Dale, 1999), o los componentes del "menú corto de política educativa” (Heyneman, 2003). El artículo realiza un ejercicio de identificación de las principales omisiones que subyacen en las tesis dominantes, e intenta subrayar las contradicciones y las relaciones inexploradas que explican otro tipo de efectos de la agenda educativa global. A efectos de presentación, estas tesis se agrupan en tres apartados, relativos a la relación entre educación y empleo, a las tesis relativas a la expansión educativa y la contención del gasto público, y a las estrategias educativas de lucha contra la pobreza. Estos tres aspectos no agotan, por supuesto, la agenda educativa global para el desarrollo, pero sí constituyen tres pilares fundamentales sobre las que se han desarrollado las políticas educativas neoliberales. 
Globalización, mercados de trabajo y educación

Los efectos de la globalización sobre el trabajo han sido uno de los campos más estudiados por las ciencias sociales (Carnoy, 2002; Castells, 1996). Las transformaciones que estos autores identifican, aún existiendo diferencias sobre su dirección y sus efectos, suponen cambios formidables en la propia organización y contenido de los trabajo y en el comportamiento de los mercados de mano de obra. Las transformaciones alcanzan también a las relaciones entre educación y trabajo, tanto en lo que se refiere a las necesidades de cualificación de los empleos, como a las retribuciones de los distintos niveles de cualificación en contextos de mercados de trabajo cada vez más interdependientes. Algunas de las tesis más destacadas en este ámbito son las siguientes:

- Las habilidades que requiere el mercado de trabajo son más generalistas y centradas en la autonomía de los sujetos (programación, inglés, habilidades de comunicación, habilidades matemáticas, resolución de problemas).

- En una economía mundial globalizada, la competitividad vendrá cada vez más determinada por la calidad de la fuerza de trabajo (a diferencia de las economías de escala propias del régimen de acumulación fordista).

- No existe movilidad de la mano de obra a escala global, pero sí interdependencia laboral que sobrepasa las fronteras (Carnoy, 2002).

- Existirá un aumento previsible de las diferencias salariales en función de las cualificaciones profesionales (especialmente entre los nuevos puestos de trabajo asociados a la innovación tecnológica y los trabajadores poco o no cualificados).

- La mayor movilidad del capital financiero e industrial repercute en las lógicas de negociación salarial en los países del norte (debilitamiento de sindicatos ante la amenaza de movilidad del capital).

Estas tesis son difícilmente discutibles como tendencias dominantes de cambio. Sin embargo, más discutible es considerar unos 
determinados efectos de estas tendencias y un conjunto de políticas consideradas adecuadas para hacerles frente. En efecto, en líneas generales, aquello que es considerado positivo para el desarrollo económico, especialmente para los países en vías de desarrollo, son las estrategias de integración y competitividad en la economía global. De este modo, la inversión estratégica en capital humano es lo que puede facilitar la atracción de capital extranjero y favorecer el desarrollo de empresas de tecnología punta. Para ello, es necesario avanzar hacia la desregulación del mercado de trabajo y crear los mecanismos necesarios para incentivar la entrada de capitales. Es la desregulación del mercado de trabajo lo que permite la generación de empleo cualificado y la mejora de la competitividad económica nacional. La inversión en empresas tecnológicas aumenta las tasas de rendimiento de la inversión en enseñanza superior y genera nuevos empleos.

La lista de políticas educativas asociadas al desarrollo económico, sin embargo, incurre en contradicciones. La desregulación de los mercados de trabajo no parece asegurar per se la generación de empleo cualificado. Existen evidencias de que uno de los principales atractivos para el capital es la reducción de costes salariales. Con la globalización, la competitividad por la reducción de costes ha alcanzado a todos los niveles de cualificación, pero no siempre con la misma intensidad y en la misma dirección. Son generalmente los trabajadores menos cualificados aquellos que más sufren la competencia salarial a escala global. En el caso de los países en vías de desarrollo (PVD), la búsqueda de la competitividad económica se ha caracterizado especialmente por políticas de liberalización de los mercados de trabajo y de de reducción del salario mínimo.

Por otra parte, la reducción o no de los salarios en niveles más altos de cualificación depende de la importancia relativa de los efectos "composición" y "compresión salarial" de los niveles de cualificación (Gundlach et al., 2001). El primer efecto tiende a aumentar la proporción de trabajadores con niveles altos de cualificación, lo que lleva a una mayor desigualdad salarial entre grupos sociales. La sobreoferta de personas cualificadas, sin embargo, puede producir un efecto de compresión salarial que reduzca las diferencias salariales entre trabajadores y, por tanto, reduzca las desigualdades de ingresos. La tendencia observable en los PVD es que el efecto composición ha sido mayor que 
el efecto compresión, una tendencia que ha ido acompañada por la reducción de los salarios reales de los niveles más bajos de cualificación.

La globalización tiene en este terreno una plasmación muy desigual entre países del norte y países de sur. La retribución netamente desigual entre ambos conjuntos de países, en todos los niveles de cualificación, acentúa la presión de la movilidad de la fuerza de trabajo a distintos niveles, incluida la "fuga de cerebros" (Vinokur, 2007). En su conjunto, estas diferencias son muy significativas si se tiene en cuenta la lógica que orienta las prioridades de inversión educativa de instituciones como el Banco Mundial. La prioridad otorgada a la inversión en enseñanza básica, que caracterizó la estrategia del Banco desde la década de los ochenta y se consolidó en el Policy Paper de 1995 (World Bank, 1995), ha sido progresivamente matizada por el propio Banco (World Bank, 2004). La globalización se reconoce como motor transformador de los rendimientos relativos de los distintos niveles de enseñanza, hasta el extremo de que las tasas de rendimiento de la enseñanza superior superan el rendimiento de la enseñanza básica. Sin embargo, son aspectos contextuales los que determinan la intensidad de ese cambio y el grado de desigualdad que generan. Paradójicamente, aquellos países en los que más evidente es el aumento del rendimiento de la educación superior son también aquellos en los que las desigualdades salariales aumentan. En los países de sur, por tanto, y a pesar de notables avances en la democratización del acceso a la enseñanza, el efecto composición parece constituirse en fuente principal de desigualdades. Persisten desigualdades sociales de acceso a la enseñanza media y superior que constituyen un claro obstáculo a la reducción de desigualdades de renta.

El contexto educativo de cada país, el grado de desigualdad de acceso a los distintos niveles de enseñanza, o la capacidad de maniobra para orientar las inversiones estratégicas de capital extranjero son algunos de los factores determinantes que pueden conducir a que los efectos de la liberalización del mercado de trabajo no se traduzcan en el desarrollo educativo necesario para el propio país.

Acerca del gasto público en educación: contener el gasto y expandir la enseñanza

La globalización sí genera un consenso prácticamente generalizado sobre las necesidades de expansión de la educación. Los cambios 
tecnológicos y la progresiva importancia de la educación como activo que condiciona la posición social y, en consecuencia, como institución clave de las oportunidades sociales, justifican la necesidad de invertir en la expansión de la enseñanza. Las políticas neoliberales, para hacer frente a las necesidades de la globalización, pasan sin embargo por la contención del gasto público. Una de las reglas del Consenso de Washington es precisamente la necesidad de contener el gasto y de no incurrir en déficit fiscal, una política estrictamente aplicada a partir de los años ochenta en diversos PVD y con resultados nefastos en el ámbito social (Reimers y Tiburcio, 1993).

La política educativa que se deriva de estos condicionantes es la de conseguir expandir el acceso a la educación sin que crezca el gasto público educativo. Dos son los mecanismos contemplados para hacerlo posible: por un lado, la expansión de la oferta privada de enseñanza; por otro lado, la redistribución interna del gasto público educativo hacia los sectores sociales más necesitados. De este modo puede conseguirse aumentar la participación en todos los niveles de enseñanza al tiempo que se aplican medidas de contención del gasto público.

La aplicación de este tipo de políticas es hoy una realidad. En muchos PVD hemos asistido al incremento de la oferta privada de educación e incluso al desarrollo de marcos regulativos que favorecen la expansión de una oferta privada homologable, sin que existan mecanismos de control de su calidad. Esta expansión ha tenido lugar especialmente en el ámbito de la enseñanza superior, pero también es visible en la enseñanza básica.

Asimismo, también han sido visibles las decisiones acerca de la redistribución interna del gasto educativo. En muchos PVD los sindicatos educativos han hecho frente a políticas de caída de los salarios reales o de precarización de las condiciones laborales del colectivo docente, como respuesta a los intentos de reducción del gasto corriente (habitualmente superior al 75\% del presupuesto educativo). La prioridad de inversión a la enseñanza básica se justifica en base a las tasas de rendimiento educativo, pero también para responder a financiar públicamente a la escolarización de los sectores más desfavorecidos socialmente. Asimismo, se han planteado también modalidades de co-pago en los niveles superiores a la enseñanza básica, bajo el argumento de que son los grupos sociales más desfavorecidos aquellos que 
más participan de la enseñanza media y superior. Los créditos educativos, especialmente en el terreno de la enseñanza superior, también son una muestra de la búsqueda de vías que aumenten el gasto privado de la enseñanza sin alterar la participación social en educación.

Las limitaciones que han encontrado estas mismas políticas para ser aplicadas con carácter estricto han conllevado en ocasiones extremar las medidas de contención del gasto educativo aún a riesgo de perjudicar a los grupos más desfavorecidos. De este modo, hemos asistido al mantenimiento o introducción del pago de tasas de escolarización en enseñanza básica (Tomasevsky, 2004), al incremento de otros costes directos de la enseñanza - material escolar, uniformes (Carnoy y Torres, 1994) -, o a procesos de empeoramiento de las condiciones de escolarización de la enseñanza pública (ejemplificada sobre todo por medio del aumento de ratios alumnos/profesor).

Cada una de las medidas, impulsadas por recomendaciones o imposiciones del Banco Mundial o por los propios gobiernos nacionales, ha sido justificada a partir de la ineludible necesidad de compatibilizar la expansión educativa con la contención del gasto. El resultado de la aplicación de estas políticas ha sido desigual en función de los países en los que se han llevado a cabo y de las especificidades de su sistema educativo. Con todo, y a la vista de algunos de sus efectos, son discutibles muchos de los supuestos sobre los que se sustentan los previsibles efectos de estas políticas. Veámoslo.

Por un lado, el aumento de la oferta privada de educación ha conllevado un inevitable aumento de las desigualdades educativas. El caso de Chile, es un ejemplo ilustrativo de ello (García Huidobro, 2006). Una legislación extremadamente permisiva con la expansión de la enseñanza privada, junto a un proceso de descentralización educativa claramente orientado hacia la reducción de costes, ha aumentado significativamente la brecha social en el acceso a las distintas redes de escolarización y en los resultados educativos. La garantía de equidad a la enseñanza privada por medio de ayudas públicas a los grupos sociales más desfavorecidos no ha tenido lugar, tanto por la escasez de dichas ayudas como por la lógica selectiva de los propios centros privados, tanto desde un punto de vista social como territorial.

La redistribución del gasto educativo interno ha sido mucho más tímida de lo necesario. En países como Brasil, la capacidad de 
resistencia de los sectores de enseñanza superior ha impedido modificar situaciones de privilegio e injusticia en la distribución del gasto público educativo. El alcance de becas o ayudas compensatorias ha sido claramente insuficiente para permitir alterar la pirámide social de acceso a la enseñanza superior. El resultado es, en muchos países, un aumento relativo de las tasas de escolarización a costa de un aumento de las desigualdades.

Por otra parte, la argumentación construida por el Banco Mundial para justificar determinadas políticas de contención del gasto, sin que repercutan en un aumento de las desigualdades educativas, ha sido claramente desmentida por la realidad. En otro lugar he cuestionado los supuestos sobre los que se sostienen los argumentos relativos a la inexistencia de costes de oportunidad en el acceso a la enseñanza primaria, a la supuesta inelasticidad-precio de la demanda educativa, o a el efecto de las percepciones acerca de la calidad de la enseñanza sobre las estrategias educativas de las familias en situación de pobreza (Bonal, 2007). El no cumplimiento de estos supuestos explica, en buena medida, los límites en la expansión educativa de los grupos más desfavorecidos o el comportamiento de indicadores como el abandono escolar prematuro.

Finalmente, existe un argumento fundamental no ponderado en las políticas educativas neoliberales. Paradójicamente, se trata de un argumento que emana del propio proceso de globalización. Me refiero a la devaluación de las credenciales educativas que se deriva de la propia expansión educativa y de la mayor movilidad de la fuerza de trabajo. El efecto de devaluación repercute en el umbral educativo mínimo necesario para escapar de la pobreza o en el comportamiento de diversos grupos sociales ante la educación. Especialmente destacable es el comportamiento de las clases medias, que, ante la caída de la calidad de la enseñanza pública, recurren a estrategias de inversión en enseñanza privada y se desentienden de defender la calidad de la enseñanza pública.

\section{Globalización, educación y pobreza}

Un tercer conjunto de argumentos relacionados con las políticas educativas neoliberales tiene relación con los efectos esperados del acceso a la educación de los niños en situación de pobreza. Es este uno 
de los componentes más importantes de las ideas que relacionan la globalización con la educación. En efecto, tanto en las reuniones y cumbres internacionales (como las impulsadas por la UNESCO en el marco del proyecto Educación para Todos, en Jomtien y Dakar, o en la cumbre de Naciones Unidas en el año 2000, que dio lugar a los "Objetivos de Desarrollo del Milenio"), como en las agendas de las agencias de desarrollo y de los propios Estados, la educación aparece como uno de los instrumentos necesarios y fundamentales para lograr la reducción y erradicación de la pobreza.

La educación es un aspecto fundamental en el desarrollo de los Documentos de Estrategia de Lucha Contra la Pobreza (DELP), impulsados por el BM y el FMI desde 1999, y que incluyen, entre otras "buenas prácticas" sectoriales, recomendaciones de inversión educativa centradas en los sectores más vulnerables (Klugman, 2002). Su elaboración es hoy un requisito indispensable para el acceso a nuevos créditos de los países altamente endeudados (HIPC). Tampoco debe sorprendernos que la educación se haya convertido en uno de los sectores prioritarios de las nuevas políticas focalizadas, que se han multiplicado especialmente en América Latina a partir de la segunda mitad de la década de 1990. Numerosos programas, dirigidos a la mejora institucional de los servicios frecuentados por los sectores pobres o diseñados a través de transferencias de renta condicionadas, concentran sus esfuerzos en el terreno educativo como mecanismo fundamental para romper la reproducción del círculo intergeneracional de la pobreza y la desigualdad.

Las propuestas políticas neoliberales en ámbito de la educación como instrumento de lucha contra la pobreza no han sido, en todo caso, estáticas. Fundamentalmente, el neoliberalismo educativo ha pasado de contemplar los efectos automáticos del crecimiento económico sobre el comportamiento educativo de las familias pobres a considerar la necesidad de desarrollar políticas educativas focalizadas que garanticen la escolarización de los niños en situación de pobreza.

En el contexto del modelo estricto del Consenso de Washington, el denominado "efecto goteo" (trickle down effect), según el cual el crecimiento económico alcanza a todos los sectores de la sociedad, se tradujo en el ámbito educativo en esperar los beneficios directos e indirectos de la expansión de la escolarización. Políticas dirigidas a 
mejorar la gestión y organización escolar y reformas educativas caracterizadas por la descentralización, la recuperación de costes y la competitividad entre escuelas fueron diseñadas para mejorar la calidad educativa a bajo coste (Pereyra et al., 1996). Las mejoras en cantidad y calidad tenían que conseguir aumentar la participación de los sectores más desfavorecidos en la enseñanza y, con ello, favorecer la movilidad social intergeneracional.

Las evidencias de los límites de la expansión educativa para reducir la pobreza son numerosas. Como señala Teresa Bracho para el caso de México, nunca la población pobre había acumulado tanto nivel educativo tan escasamente intercambiable en el mercado de trabajo (Bracho, 2002). Los argumentos anteriormente expuestos sobre la devaluación de las credenciales educativas pueden considerarse especialmente pertinentes para comprender los límites de la expansión educativa para reducir la pobreza. Por otra parte, las reformas educativas referidas han consolidado distintas redes de calidad educativa, siendo las de menor calidad las que son habitualmente frecuentadas por los sectores pobres. Cabe destacar también que las políticas educativas neoliberales han tendido a minimizar el efecto de las necesidades derivadas de la crisis y de la mala calidad educativa sobre las estrategias de inversión educativa de las familias pobres (Bonal, 2007).

La dureza del ajuste se proyectó también, por lo tanto, en el ámbito educativo, con costes significativos en las desigualdades de acceso y de participación en los distintos niveles de enseñanza y en el abandono escolar.

A ello reaccionó el Banco Mundial, que a partir de finales de los años de 1990 contempla la necesidad de invertir directamente en los sectores pobres para corregir las imperfecciones de un modelo que se considera el adecuado, pero que no consigue "activar" a la población pobre. El informe de 2004 es el mejor exponente de la nueva visión del Banco en su misión de lucha contra la pobreza (World Bank, 2004). En el terreno educativo, las nuevas políticas educativas para atender a las necesidades de la población pobre pasan por los mecanismos de focalización.

En efecto, la emergencia y expansión de los programas de intervención social focalizada hay que situarlas en la segunda mitad de la década de 1990, dentro de las denominadas reformas de segunda 
generación. El fracaso de los Programas de Ajuste Estructural y sus dramáticos costes sociales desencadenaron importantes críticas al FMI y al Banco Mundial, que reclamaron nuevas políticas con "rostro humano". Las instituciones de Bretton Woods, por su parte, respondieron a las críticas desde principios de la década de 1990 con discursos y políticas que las erigieran como instituciones globales de lucha contra la pobreza. Identificar a los pobres y concentrar recursos y esfuerzos en proporcionarles posibilidades de acceso a los distintos mercados se convirtieron en la estrategia política dominante. La nueva estrategia repercutió rápidamente en la cartera de préstamos del Banco Mundial y del FMI, de modo que al final de la década de 1990 los denominados "fondos sociales de emergencia" alcanzaron el 50\% de los créditos de ajuste estructural (Mundy, 2002, p. 492).

La aplicación de los programas focalizados en educación ha tenido efectos dispares en función de los programas y de los contextos en los que han sido aplicados. Programas de transferencia de renta condicionados a la asistencia escolar, como el Bolsa Escola de Brasil o el Oportunidades en México, han conseguido aumentar la participación de niños en situación de pobreza en las escuelas. Las evaluaciones también arrojan limitaciones importantes, desde el punto de vista de los resultados educativos o del aprovechamiento de la experiencia escolar de los niños en situación de pobreza (Bonal y Tarabini, 2006a). Una de las paradojas de las políticas focalizadas es que, para ser eficaces, requieren un grado de extensión e intensidad muy alto, hasta el punto de que pueden llegar a dejar de tener sentido como políticas focalizadas y parecerse más a políticas universalistas. Los problemas presupuestarios y los distintos contextos de pobreza, por otra parte, han repercutido sobre diferencias muy significativa en la intensidad de las transferencias, con lo que la eficacia de los programas ha sido muy desigual (Bonal y Tarabini, 2006b).

Pero aquello que explica más claramente los límites de las políticas educativas focalizadas para luchar contra la pobreza es su carácter casi exclusivamente monetario. Unas formas de experimentar la pobreza que va mucho más allá de lo material explican que, en muchas ocasiones, falten estrategias que compensen los numerosos obstáculos que encuentran los niños pobres para hacer frente a una experiencia escolar con éxito. De ello se desprende que ninguna política educativa puede ser efectiva como estrategia de lucha contra la pobreza, si no tiene en 
cuenta los efectos de la pobreza sobre la educación. Esta omisión, persistente en las diferentes aproximaciones de política educativa, no sólo relativiza la centralidad de la educación como mecanismo de ruptura de la pobreza intergeneracional, sino que explica por qué en tantas ocasiones políticas diseñadas para los colectivos más desfavorecidos presentan unos pobres resultados. Las políticas sociales pensadas para la "activación" de los pobres sitúan a menudo en el terreno de la "privación cultural" las necesidades de intervención, al tiempo que ignoran un conjunto de impedimentos objetivos que limitan las posibilidades reales para que los pobres desarrollen inversiones sostenibles, como es la propia inversión en capital humano. Cuando sí existe el reconocimiento a estos impedimentos, por otra parte, se recurre a las estrategias de facilitar el acceso a unos mercados que deben adaptarse a las condiciones sociales y económicas de los grupos excluidos (World Bank, 2004), ignorando por completo los propios límites de cualquier mercado para adaptarse a las necesidades de los pobres y los límites de los pobres para subsistir a través de su acceso a mercados formales. El discurso sobre la "activación" de los pobres, asimismo, choca frontalmente con el desarrollo de unas prácticas selectivistas, como son las que se derivan de la focalización. Debido a sus limitaciones de cobertura, los programas focalizados alcanzan fundamentalmente a aquellos colectivos que, por la intensidad de su pobreza, difícilmente consiguen ser "activados" y sólo temporalmente asistidos.

Esta cuestión sitúa en primer plano la necesidad de servirse de la noción de "educabilidad" para desarrollar políticas que efectivamente contemplen no sólo los factores materiales de la pobreza. La educabilidad puede ser definida como el conjunto de condiciones materiales, sociales, culturales y emocionales necesarias para el aprendizaje (López, 2004; López y Tedesco, 2002). La consideración de la educabilidad constituye un elemento muy positivo en la valoración de la relación entre educación y pobreza, puesto que pone el énfasis precisamente en aquellos factores asociados a la pobreza que impiden el aprovechamiento de las oportunidades educativas. Supone, por lo tanto, el tener en cuenta muchas de las omisiones señaladas a lo largo de este texto y permite mostrar los límites de las reformas institucionales para conseguir el éxito educativo de los grupos más excluidos, así como la necesidad de desarrollar estrategias multisectoriales y multidimensionales de lucha contra la pobreza. Supone también considerar que la 
pobreza no es sólo material, y que existen otros impedimentos para el éxito escolar. La consecución de las condiciones de educabilidad requiere también de la implicación colectiva para hacerse efectiva: del Estado, de las familias, de la escuela y de la sociedad civil. Esta implicación colectiva añade la necesaria corresponsabilidad que necesita la acción educativa en beneficio de los sectores socialmente excluidos y abre la posibilidad de poder interrogarse si la escuela que reciben los pobres es realmente la escuela que necesitan.

La experiencia nos demuestra que es más fácil expandir el acceso que expandir el aprendizaje, y que el esfuerzo material y humano que requiere éste supera ampliamente el primero, sobre todo, cuando de lo que se trata es de conseguir que un alumnado con carencias de todo tipo consiga no sólo acceder a la escuela, sino aprovechar su paso por ella, de modo que consiga romper la reproducción de la situación social de su familia. La educación pasa, de este modo, del área blanda al área dura de la política social, donde la consecución de los objetivos requiere de mayores esfuerzos y se enfrenta a situaciones de mayor incertidumbre.

Poner énfasis en el concepto de educabilidad permite adentrarse en las diversas formas en las que la experimentación de la pobreza puede influir sobre el habitus de niños y niñas ante la educación, permite llamar la atención sobre la importancia de explorar las distintas culturas de la pobreza, permite preguntarse hasta qué punto la organización escolar, el currículum o la pedagogía tienen en cuenta las condiciones sociales, económicas y afectivas del alumnado pobre y permite, en definitiva, planificar una intervención educativa que tenga en cuenta las diversas formas en que la propia pobreza dispone a los individuos ante la educación.

\section{A modo de conclusión: globalización, educación y no beneficiarios}

Este artículo ha tratado de identificar un conjunto de tesis presentes en la agenda educativa global, tesis que han formado parte en gran medida de las aproximaciones neoliberales al desarrollo y que, con diversas formas y matices, han tenido una clara traducción empírica en muchos países, tanto económicamente avanzados como, especialmente, en vías de desarrollo. El texto ha identificado las bases sobre 
las que estas tesis se construyen y, sobre todo, los efectos previstos sobre el desarrollo educativo esperado de su aplicación.

La reducción a unas relaciones causa-efecto entre la política educativa y sus beneficios previsibles sitúa el discurso neoliberal sobre el desarrollo educativo como construcción eficaz para favorecer las oportunidades educativas de los sectores más desfavorecidos. Sin embargo, la revisión de los resultados de la aplicación de estas políticas arroja un balance que desmiente en gran medida la supuesta eficacia de las mismas. Ni desde el lado de la oferta, ni desde el lado de la demanda, las instituciones y los agentes educativos han respondido a las expectativas del "menú corto de política educativa". Los efectos "no esperados" han sido especialmente visibles en los países en desarrollo: la expansión de la educación no parece haber constituido el motor de desarrollo fundamental del crecimiento económico y menos aún el mecanismo de igualación de oportunidades sociales y de reducción de la pobreza y la desigualdad.

Es interesante constatar que el cuestionamiento de la eficacia de las políticas educativas hegemónicas no es una consecuencia directa de los cambios que conlleva la globalización, sino de las políticas que se han considerado pertinentes para responder a sus retos. El artículo ha intentado identificar las omisiones en las relaciones causa-efecto de las políticas educativas y evidencias sobre los efectos indirectos o no esperados de la aplicación de las mismas.

No hay duda de que existen beneficios derivados de la agenda educativa global para el desarrollo. Pero es igualmente visible el hecho de que ni los beneficios se han distribuido de forma equitativa entre grupos sociales, ni los sectores más desfavorecidos han sido quienes hayan obtenido una mayor ventaja comparativa. Este hecho es especialmente relevante en la medida en que las posiciones de partida de los grupos sociales son desiguales y son precisamente los grupos menos favorecidos los que deberían experimentar un mayor progreso, tanto en el acceso a la educación, como en los resultados educativos. Solamente a partir de la aplicación de políticas educativas focalizadas se han podido observar avances en la situación educativa de los colectivos más vulnerables. Las limitaciones, sin embargo, como se ha expuesto anteriormente, son muchas para que la concentración de recursos constituya un mecanismo eficaz para proveer oportunidades educativas suficientes. 
La consideración de los efectos no esperados de la aplicación de políticas es un aspecto fundamental para la revisión de la agenda educativa global para el desarrollo. Para ello son necesarias evaluaciones que se desmarquen de los modelos rígidos que pre-establecen las variables e indicadores a observar y que pueden presentar importantes sesgos sobre la valoración de la eficacia de las políticas educativas.

Recebido em julho de 2009 e aprovado em agosto de 2009.

\section{Referencias}

BONAL, X. On global absences: reflections on the failings of the education and poverty relationship. International Journal of Educational Development, v. 27, n. 1, p. 86-100, 2007.

BONAL, X.; TARABINI, A. Programas de garantía de renta condicionados a la asistencia escolar. Una revisión de evaluaciones del programa Bolsa Escola. Revista Mexicana de Investigación Educativa, México, DF, v. 11, n. 31, oct.-dic. 2006a.

BONAL, X.; TARABINI, A. Focalización educativa y lucha contra la pobreza: una discusión acerca de los límites y posibilidades del programa Bolsa Escola. In: Bonal, X. (Ed.). Globalización, educación y pobreza en América Latina: ¿hacia una nueva agenda política? Barcelona: CIDOB, 2006b.

BRACHO, T. Educación y pobreza en México, 1984-1996. In: ReImers, F. (Ed.) Distintas escuelas, diferentes oportunidades: los retos para la igualdad de oportunidades en Latinoamérica. Madrid: La Muralla, 2002.

CARNOY, M. Sustaining the new economy: work, family, and community in the information age. New York: Russell Sage Foundation, 2002.

CARNOY, M.; TORRES, C.A. Educational change and structural adjustment: a case study of Costa Rica. In: SAMOFF, J. (Ed.). Coping with crisis: austerity, adjustment and human resources. London: Cassell, 1994. 
CASTELLS, M. La era de la información: economía, sociedad y cultura. Madrid: Alianza, 1996. v.1.

FINE, B. Social capital versus social theory. London: Routledge, 2001.

GARCÍA HUIDOBRO, E. La reforma educacional chilena y la educación pública. In: BONAL, X. (Ed.). Globalización, educación y pobreza en América Latina: ¿hacia una nueva agenda política? Barcelona: CIDOB, 2006.

GUNDLACH, E.; DE PABLO, J.N.; WEISERT, N. Education is good for the poor. World Institute for Development Economics Research, Discussion paper n. 137, 2001. Disponível em: <http://www.wider. unu.edu/publications/dps/dp2001-137.pdf>. Acesso em: 15 jul. 2007.

DALE, R. Specifying globalisation effects on national policy: focus on the mechanisms. Journal of Education Policy, London, v. 14, p. 1-17, 1999.

HEYNEMAN, S.P. The history and problems in the making of education policy at the World Bank 1960-2000. International Journal of Educational Development, n. 23, p. 315-337, 2003.

KLUGMAN, J. Estrategias de reducción de la pobreza: introducción. Washington, DC: World Bank, 2002.

LÓPEZ, N. Educación y equidad: algunos aportes desde la noción de educabilidad. Buenos Aires: IIPE, 2004.

LÓPEZ, N.; TEDESCO, J.C. Las condiciones de educabilidad de los niños y adolescentes en América Latina. Buenos Aires: IIPE, 2002.

MUNDY, K. Retrospect and prospect: education in a reforming World Bank. International Journal of Educational Development, n. 22, p. 483-508, 2002.

PEREYRA, M. et al. Globalización y descentralización de los sistemas educativos. Barcelona: Pomares-Corredor, 1996.

REIMERS, F.; TIBURCIO, L. Education, adjustment and reconstruction: options for change. Paris: UNESCO, 1993. 
ROBERTSON, S.; BONAL, X.; DALE, R. GATS and the education service industry: the politics of scale and global re-territorialization. Comparative Education Review, Chicago, v. 46, n. 2, p. 472-496, 2002.

TOMASEVSKY, K. Los derechos económicos, sociales y culturales: el derecho a la educación. [S.1.]: Naciones Unidas; Consejo Económico y Social, 2004.

VERGER, A. L'AGCS i la politica de l'educación. 2008. Tese (Doutorado) - Departamento de Sociología, Universitat Autónoma de Barcelona, Bellaterra.

VINOKUR, A. El debate de la globalización y la fuga de cerebros. In: Bonal, X.; Tarabini, A.; Verger, A. (Ed.). Globalización y educación. Buenos Aires: Miño y Dávila, 2007.

WORLD BANK. Policies and strategies for education: a World Bank review. Washington, DC: World Bank, 1995.

WORLD BANK. World Development Report 2004: making services work for poor people. Washington, DC: World Bank, 2004. 